\title{
A Census of Brown Dwarf Binaries
}

\author{
Wolfgang Brandner and Hervé Bouy \\ European Southern Observatory, Karl-Schwarzschild-Straße 2, D-85748 \\ Garching, Germany
}

\begin{abstract}
Brown dwarf binary systems offer the unique opportunity to measure some of the underlying physical characteristics, like, e.g., the mass. In addition, the individual components of brown dwarf binaries are expected to be coeval and have the same chemical composition. This provides crucial constraints on any (evolutionary) model by greatly reducing the number of free parameters. Finally, brown dwarf binary statistics should shed light on the origin of brown dwarfs and possible formation mechanisms and environments. We summarize on-going surveys for brown dwarf binaries, and present first results of follow-up studies.
\end{abstract}

\section{Benefits of Brown Dwarf Binary Studies}

The study of brown dwarf binaries offers a multitude of benefits: (1) A search for companions to known brown dwarfs is one of the best ways to identify objects of still lower mass and effective temperature. (2) Long-term studies of brown dwarf binary orbits will ultimately lead to dynamical mass estimates from orbital parameters. (3) Studies of the (co-eval) individual components of brown dwarf binary systems can be used to test evolutionary track and atmospheric models (same chemical composition). (4) The properties of brown dwarf binaries yield clues on the origin of (binary) brown dwarfs. Thus there is a clear need for systematic surveys for brown dwarf binaries.

\section{Surveys for Brown Dwarf Binaries}

\subsection{Spectroscopic Brown Dwarf Binaries}

PPl 15, the first brown dwarf in the Pleiades spectroscopically confirmed by the presence of lithium (Basri et al. 1996), incidentally also turned out to be the first spectroscopic binary brown dwarf to be identified (Basri \& Martín 1999). In the case of SB2s (like PPl 15), the mass-ratio of the components can be derived directly.

A survey for spectroscopic binaries among young brown dwarfs in Chamaeleon was carried out by Joergens \& Guenther (2001, see also contribution in these proceedings). Five out of twelve young brown dwarfs in Chamaeleon exhibit radial velocity variations, which could be either due to surface features (spots) or companions. 


\subsection{Visual Brown Dwarf Binaries}

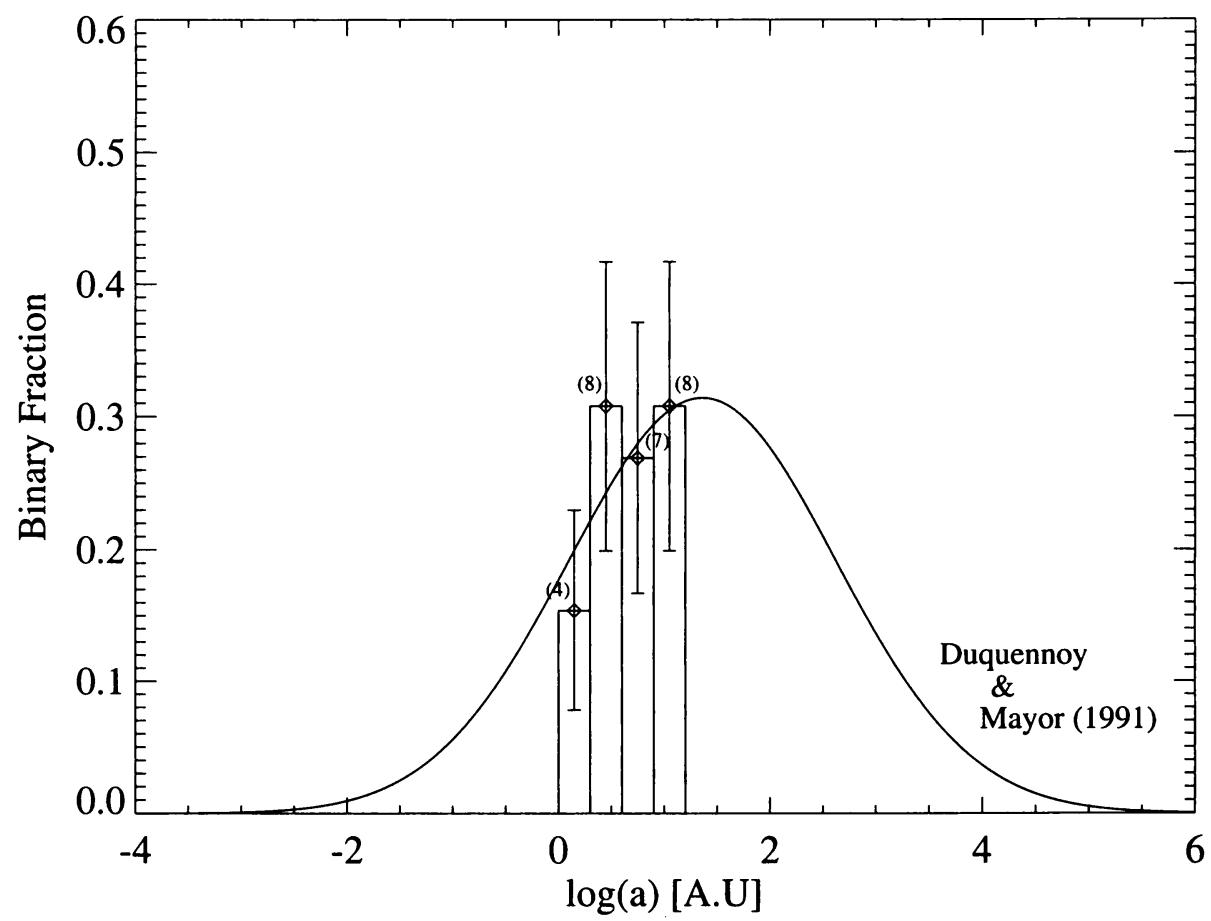

Figure 1. Distribution of separations for field binary ultracool dwarfs. The solid curve represents the model distribution of Duquennoy \& Mayor (1991) for G stars. The lack of ultracool dwarf binaries at small separations is due to the limited spatial resolution ( 2.0 A.U.) of present-day surveys. The absence of ultracool dwarf binaries with large separations is real.

Surveys for visual (i.e., spatially resolved) brown dwarf binaries require high angular resolution. This can be achieved by using ground-based telescopes in combination with adaptive optics, or from space using HST.

The first systematic survey for binaries among brown dwarfs in the Pleiades was carried out by Martín et al. (2000) using HST/NICMOS and CFHT/PUEO. No companions at projected separations larger than $27 \mathrm{~A} . \mathrm{U}$. were found among 30 very-low mass stars and brown dwarfs. Another imaging survey for companions to the 12 young brown dwarfs in Chamaeleon using HST/WFPC2 also yielded negative results, i.e., no companion was found at projected separations larger than 30 A.U. (Neuhäuser et al. 2002). Among nearby star forming regions, only IC 348 yielded one candidate brown dwarf binary with a separation of 2500 A.U. (Najita et al. 2000, see however also contribution by Luhman, these proceedings).

Similarly, the sky surveys DENIS, 2MASS and SDSS did not find any resolved L- or T-dwarf binaries (see contributions by Forveille, Kirkpatrick and Covey). 
Hence high angular resolution alone is not sufficient to detect companions to brown dwarfs. The first spatially resolved brown dwarf binary was found among the free-floating brown dwarfs in the solar neighbourhood. Martín et al. (1999) used HST/NICMOS to resolve DENIS 1228.2-1547. Subsequently, many more binaries could be resolved among the free-floating brown dwarfs (Koerner et al. 1999, Reid et al. 2001, Close et al. 2002, and contributions to these proceedings).

Up to now, at least 26 spatially resolved binaries have been identified among nearby ultracool dwarfs and brown dwarfs (see Bouy et al. 2002).

\section{Census and Physical Properties}

Figure 1 shows the distribution of separations for ultracool dwarf and brown dwarf binaries. Binaries with separations larger than 15 A.U. are absent.

Figure 1 of accompanying paper in this volume by Bouy et al. shows brightness difference between brown dwarf binary components vs. separation. There is a strong preference for brightness differences $\leq 1 \mathrm{mag}$, indicative that most binaries have mass ratios close to unity.

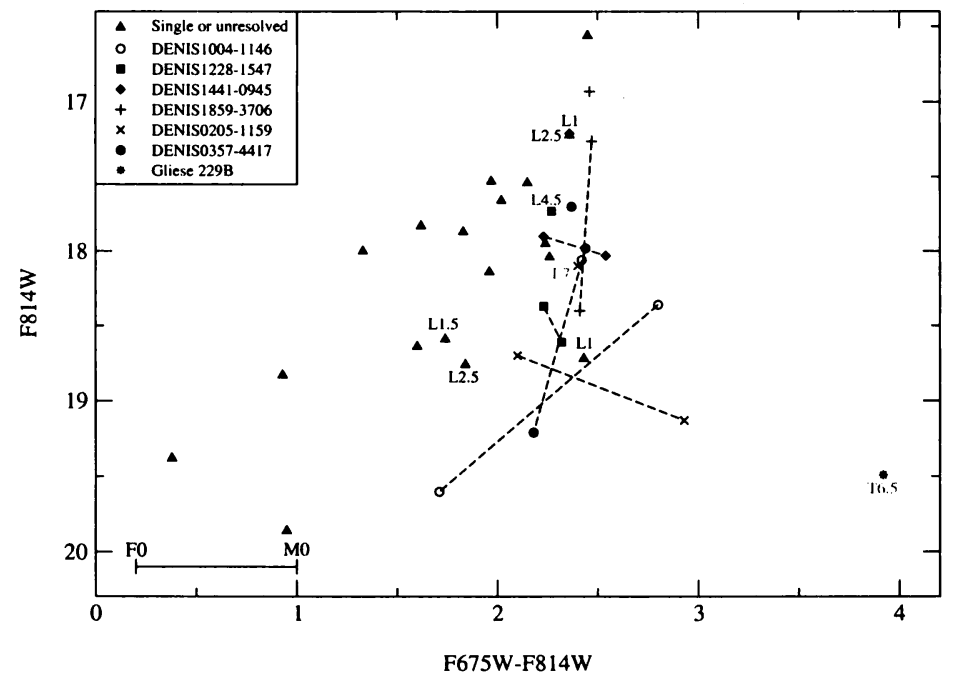

Figure 2. Colour Magnitude diagram for brown dwarfs. Single or unresolved objects are represented by filled triangles (down). Binary systems are represented by various symbols. For each binary system, both the binary system and the two components are represented (same symbol), these latter two are joined by a dashed line. The T-dwarf G1229B is represented (by an asterisk) in addition for comparison. Values for Gl229B from Golimowski et al. (1998). 
Figure 2 shows a colour-magnitude diagram for brown dwarfs as observed with HST/WFPC2. Note that the secondary (fainter component) in a brown dwarf binary system can be either redder or bluer than the primary.

The properties of the binaries might be indicative of the formations process and/or the origin of brown dwarfs.

\section{The Origin of (binary) Brown Dwarfs}

Clarke (1996) summarized the possible formation modes for binaries. For brown dwarf binaries, the most likely scenarios appear to be the formation in small$\mathrm{N}$ clusters or the formation in protostellar disks, of which close brown dwarf binaries might get ejected (see contributions by Boss, Bate, Sterzik). Recently, Reipurth \& Clarke (2001) proposed that brown dwarfs might originate as ejected stellar embryos. In this model, a prospective protostar in a chaotic multiple system gets ejected before its accreted mass has passed the substellar/stellar boundary. While promising to explain at least a certain fraction of the brown dwarfs seen in star forming regions, it remains to be seen if the ejected stellar embryo model is capable of reproducing the binary properties observed among free-floating brown dwarfs.

\section{References}

Basri, G., Martín, E.L. 1999, AJ 118, 2460

Basri, G., Marcy, G. W., Graham, J.R. 1996, ApJ, 458, 600

Bouy, H., Brandner, W., Martín, E.L. et al. 2002, subm. to AJ

Clarke, C.J., in "NATO ASI Evolutionary Processes in Binary stars', Evolutionary Processes in Binary Stars, 1996, eds. RAMJ Wijers, MB Davies and CA Tout, Kluwer, p. 31

Close, L.M., Siegler, N., Potter, D. et al. 2002, ApJ 567, L53

Duquennoy, A., Mayor, M. 1991, A\&A 248, 485

Golimowski, D.A., Burrows, C.J., Kulkarni, S.R. et al. 1998, AJ 115, 2579

Joergens, V., Guenther, E. 2001, A\&A 379, L9

Koerner, D.W., Kirkpatrick, J.D., McElwain, M.W., Bonaventura, N.R. 1999, ApJ 526, L25

Martín, E.L., Brandner, W., Basri, G. 1999, Science 283, 1718

Martín, E.L., Brandner, W., Bouvier, J. et al. 2000, ApJ 543, 299

Najita, J.R., Tiede, G.P., Carr, J.S. 2000, ApJ 541, 977

Neuhäuser, R., Brandner, W., Alves, J. et al. 2002, A\&A 384, 999

Reid, I.N., Gizis, J.E., Kirkpatrick, J.D., Koerner, D.W. 2001, AJ 121, 489

Reipurth, B., Clarke, C. 2001, AJ, 122, 432 